\title{
THE IMPACT OF SYSTEMIC INFLAMMATION ON ANEMIA IN PATIENTS WITH CHRONIC OBSTRUCTIVE PULMONARY DISEASE
}

DOI: 10.36740/WLek202002123

Anna V. Kovchun, Vladyslav A. Smiianov, Nataliia G. Kuchma, Vladyslava V. Kachkovska, Lyudmyla N. Prystupa

SUMY STATE UNIVERSITY, SUMY, UKRAINE

\begin{abstract}
The aim of our work was to study the indicators of systemic inflammation in patients with chronic obstructive pulmonary disease (COPD) with anemia of chronic disease (AHD). Materials and methods: The study included 144 COPD patients (1 group) without anemia (hemoglobin $>120 \mathrm{~g} / \mathrm{l}$ for women and $>130 \mathrm{~g} / \mathrm{l}$ for men), and $33 \mathrm{patients} \mathrm{(2}$ group) with COPD and ACD (hemoglobin $<120 \mathrm{~g} / \mathrm{l}$ for women and $<130 \mathrm{~g} / \mathrm{l}$ for men, soluble transferrin receptors (sTFR) $-8.7-28.1 \mathrm{nmol} / \mathrm{l}$ ). The control group included 62 practically healthy people. All patients were evaluated for the content of ferritin, C-reactive protein (C-RP) and hepcidin. Statistical processing of the results was performed by using the SPSS-21 program.

Results: Patients with COPD and ACD have a significantly higher ferritin level $(p<0.001)$ compared to COPD patients without anemia and patients in the control group. The content of $C-R P$ and hepcidin in patients with COPD and ACD is also significantly $(p<0.001)$ higher compared to patients without anemia and patients in the control group. Conclusions: Determination of the content of ferritin, C-RP and hepcidin in patients with COPD may allow adequate treatment for this group of patients.
\end{abstract}

KEY WORDS: chronic obstructive pulmonary disease, inflammation, anemia of chronic disease, C-reactive protein, hepcidin

\section{INTRODUCTION}

Over the past century, new data appeared on the pathogenetic mechanisms of the development and course of COPD, as well as other clinical and laboratory manifestations, which previously seemed to be not related to the pulmonary pathology present in a patient. The development of clinical and fundamental medicine made it possible to distinguish various sets of symptoms (clinical and laboratory), which initially were hardly associated with the underlying disease, and which were rather interpreted as comorbidity. However, as the studies on COPD increased, the pathogenetic relationships between COPD and some clinical and laboratory syndromes became more clear and obvious. In this regard, most extrapulmonary manifestations are now interpreted as systemic effects of COPD [9]. The data obtained were eventually reflected in 2018 Global Strategy for the Diagnosis, Management, and Prevention of COPD [5]. It contains an indication of the systemic nature of this broncho-pulmonary pathology: "Morbidity from COPD may be affected by other concomitant chronic conditions (e.g., cardiovascular disease, musculoskeletal impairment, diabetes mellitus) related to COPD. These chronic conditions may significantly impair patient's health status, in addition to interfering with COPD management." The above is confirmed by the characteristics of COPD systemic manifestations: "Inflammatory mediators in the circulation may contribute to skeletal muscle wasting and cachexia, and may initiate or worsen comorbidities such as ischemic heart disease, heart failure, osteoporosis, normocytic anemia, diabetes, depression and metabolic syndrome."
Recent epidemiological studies show that $17-24 \%$ of patients with COPD have anemia and only 6-10\% - have polycythemia $[1,4,11]$. The pathogenesis of anemia in patients with COPD is extremely complex and poorly understood. It has been established that hepcidin plays the role of a universal humoral regulator of iron $(\mathrm{Fe})$ metabolism. It is proved that under the influence of pro-inflammatory cytokines, namely IL-6, IL-8, TNF- $\alpha$, as well as acute phase proteins (C-reactive protein (C-RP), fibrinogen), hepcidin is overproduced by the liver and blocks the receptors of ferroportin, a transmembrane protein that mediates iron release from cells (enterocytes, macrophages, hepatocytes) [3]. Hepcidin enhances iron redistribution from the bone marrow to macrophages and thus leads to impaired iron metabolism via increased iron accumulation in the cells of the macrophage system and impaired utilization of iron by erythroid cells for hemoglobin formation (iron-redistributing mechanism of anemia). At the same time, under the influence of cytokines, proliferation of erythroid precursor cells gets impaired and the erythrocyte life time shortens, which is probably associated with increased activity of the reticuloendothelial system and increased phagocytosis, as well as decreased expression of erythropoietin receptors [13]. In clinical studies, IL-10 proved to lead to anemia and increased ferritin content, reducing the availability of iron for red blood cell precursors [16]. IL-1 $\beta$ increased ferritin synthesis without increasing transcription or transcription receptor that is not associated with new iron intake. As a result, the authors suggested that IL- $1 \beta$ can lead to creation of direct intracellular iron repository, which cannot be used for hemoglobin synthesis [14]. 
Taking into account that studies of anemia of chronic disorder or, as it is also referred to, anemia of chronic disease (ACD) in patients with COPD are fragmentary, but most authors underline the key role of the chronic inflammatory process in the development of the anemia, further study of systemic inflammation markers is relevant in this category of patients.

\section{THE AIM}

The objective of our work was to study the levels of systemic inflammation markers in patients with COPD, depending on the presence or absence of ACD.

\section{MATERIALS AND METHODS}

We examined 202 patients with COPD, of which 144 patients had no anemia, 33 patients had ACD, 12 - had erythrocytosis, and 13 - had iron deficiency anemia (IDA). COPD was diagnosed according to the recommendations of GOLD 2014-2019, the diagnosis of anemia was made according to the recommendations of WHO: $\mathrm{Hb}<120 \mathrm{~g} / \mathrm{L}$ for women and $<130 \mathrm{~g} / \mathrm{L}$ for men, ACD was diagnosed at the level of soluble transferrin receptors (sTfR) 8.7-28.1 $\mathrm{nmol} / \mathrm{L}$, IDA was diagnosed at sTfR $>28.1 \mathrm{nmol} / \mathrm{L}$. For further analysis of systemic inflammation markers in patients with COPD and ACD, we formed two groups: Group I included 144 patients without anemia, Group II consisted of 33 COPD patients with ACD. Patients were enrolled in the study after informed consent was obtained in accordance with the Helsinki Declaration of the World Medical Association on the Ethical Principles of Scientific and Medical Research. The study was approved by the Bioethics Committee for experimental and clinical studies at Sumy State University Medical Institute (protocol No. 2/1 of February 14, 2017). Inclusion criteria were: age 40 years and older, diagnosis of COPD. Exclusion criteria were: severe comorbid conditions (pulmonary tuberculosis, oncopathology, alcohol and/or drug abuse, AIDS, stage II or III heart failure, as well as decompensated hepatic or renal failure), known source of bleeding (complications of peptic ulcer, chronic ulcerative colitis, chronic hemorrhoids, etc.), previously diagnosed true anemias (megaloblastic, aplastic, hemolytic), use of ACE inhibitors, pregnancy or lactation, chronic administration of systemic GCs. The control group consisted of 62 apparently healthy individuals. Ferritin content was measured by latex-enhanced immunoturbidimetric assay using Roche reagent kits (Germany) on a Cobas c311 automatic analyzer (Japan). Serum CRP level was measured by the immunoturbidimetric assay using the Dialab kit (Austria) according to the manufacturer's guidelines. Serum hepcidin level was measured by solid-phase enzyme immunoassay (ELISA) on Labsystems automated immunoassay analyzer, Multiscan MS (Finland) using Peninsula Laboratories International kit, Cat. No. S-1337, Hepcidin-25 (human) (USA) according to the manufacturer's guidelines. Statistical data were processed using SPSS-21 program.

\section{RESULTS AND DISCUSSION}

The results of the analysis of inflammation markers content, namely ferritin, CRP, and hepcidin, are presented in Table 1.

It was found that patients with COPD and ACD had a higher level of ferritin compared with patients without anemia. Higher ferritin content can indicate a high level of iron pool and excludes iron deficiency, as the cause of this anemia. On the other hand, ferritin is an acute phase protein; therefore, its high content may be attributed to an active inflammatory process [2]. Along with high content of ferritin, high levels of CRP were observed in patients with COPD and ACD, which may also indicate the leading role of the inflammatory process as a cause of ACD in patients with COPD. Our analysis of the content of hepcidin, a protein that is the main regulator of iron content, also showed a higher level of it in patients with COPD and ACD compared with patients without anemia, which may indicate a connection between the development of anemia and increased concentration of hepcidin. In order to determine, whether there is an association between hemoglobin level and inflammatory markers concentration, we carried out a correlation analysis between these parameters. A significant negative correlation was found between hemoglobin and hepcidin $(r=-0.794 ; \mathrm{p}<0.001)$, between hemoglobin and ferritin $(\mathrm{r}=-0.177 ; \mathrm{p}=0.001)$, between hemoglobin and CRP ( $\mathrm{r}=-0.744 ; \mathrm{p}<0.001)$, which may indicate an association between the levels of inflammation markers and hemoglobin in patients with COPD.

According to the results of our study, patients with COPD and ACD have significantly higher ferritin content compared with patients with COPD without anemia and patients in the control group, which is consistent with the results of an earlier study [17]. According to the data of this study, ferritin can be used in the complex differential diagnosis of iron deficiency anemia and ACD, since its levels are normal to elevated in patients with ACD and are significantly reduced in patients with iron deficiency anemia. It should be noted that ferritin is a marker of inflammation, and also performs the function of iron storage in a soluble form, which can explain its high content in patients with COPD and ACD. These data add to the results of other authors who found that IL-10 and IL- $1 \beta$ as pro-inflammatory cytokines contribute to an increase in ferritin content $[13,15]$. According to literature sources, patients with COPD and anemia show a high level of CRP and IL-6 [7]. Similar results were observed in our study, that is, the content of CRP and hepcidin in the studied patients with COPD and ACD was also significantly higher compared with patients without anemia and the control group. A higher content of CRP in patients with ACD compared with patients without anemia may indicate an active and pronounced inflammatory process in the group of patients with COPD and ACD. Our results are consistent with the results of German researchers who found that in patients with ACD, increased serum prohepcidine level is associated with increased storage of ferritin in monocytes [14]. Nemeth E. et al. found that hepcidin mRNA expression 
Table 1. The content of ferritin, C-reactive protein, and hepcidin in patients with chronic obstructive pulmonary disease, depending on anemia status

\begin{tabular}{|c|c|c|c|c|c|c|c|}
\hline \multirow{2}{*}{\multicolumn{2}{|c|}{ Parameter }} & \multicolumn{6}{|c|}{ Group } \\
\hline & & $\begin{array}{c}1 \\
n=144\end{array}$ & $\mathbf{P}_{1}$ & $\begin{array}{c}2 \\
n=33\end{array}$ & $\mathbf{P}_{2}$ & $\mathbf{P}_{3}$ & $\begin{array}{c}\text { Control group } \\
n=62\end{array}$ \\
\hline \multirow{2}{*}{$\begin{array}{c}\text { Ferritin, } \\
\mathrm{mcg} / \mathrm{L}\end{array}$} & $\mathrm{m}$ & $259.04 \pm 0.81$ & 0.001 & $475.8 \pm 2.23$ & 0.954 & 0.001 & $262.02 \pm 3.38$ \\
\hline & $f$ & $106.02 \pm 1.21$ & 0.001 & $203.5 \pm 3.5$ & 1.0 & 0.001 & $104.99 \pm 1.51$ \\
\hline \multicolumn{2}{|c|}{$\mathrm{CRP}, \mathrm{mg} / \mathrm{dL}$} & $1.56 \pm 0.2$ & 0.001 & $2.97 \pm 0.5$ & 0.001 & 0.001 & $0.476 \pm 0.14$ \\
\hline \multicolumn{2}{|c|}{ Hepcidin, ng/mL } & $21.4 \pm 0.12$ & 0.001 & $32.07 \pm 0.36$ & 0.001 & 0.001 & $13.62 \pm 2.29$ \\
\hline
\end{tabular}

Notes: $P_{1}$ - statistical significance between Group I and Group II;

$\mathrm{P}_{2}$ - statistical significance between Group I and the control group;

$\mathrm{P}_{3}^{2}$ - statistical significance between Group II and the control group.

can get increased simultaneously with an increase in serum ferritin and IL-6 levels [10], and the results of our study demonstrate a simultaneous increase in serum ferritin, CRP, and hepcidin in patients with COPD, whereas patients with COPD and ACD have significantly higher concentrations of the presented markers of inflammation. Recent experimental study by Kemna E. et al. presented evaluation and demonstrated the dynamic pattern for inflammation markers and, as a result, changes in serum iron content under the influence of lipopolysaccharides. It was found that administration of lipopolysaccharides increases IL-6 level after 3 hours, urine hepcidin level - after 6 hours, CRP level - after 12 hours, and reduces serum iron level after 22 hours by $12 \mathrm{mmol} / \mathrm{L}$ [8]. Data on administration of IL-6 in patients with Hodgkin's lymphoma add to the information on the role of pro-inflammatory cytokines and hepcidin in the development of ACD: a rapid increase in urine hepcidin was found with its further recovering within 24 hours. It is important to note that 2 hours after the infusion, serum iron level decreased by $34 \%$, and transferrin saturation decreased by $33 \%$ [6]. The results of our study are consistent with those obtained in the study of Parveen S. et al. - the authors found a negative correlation between hemoglobin and CRP in patients with COPD [11]. Thus, we conducted a comprehensive study of systemic inflammation markers in patients with COPD, as well as of the correlation between inflammatory markers level and hemoglobin concentration in patients with COPD.

\section{CONCLUSIONS}

Patients with COPD and ACD had significantly higher levels of ferritin, CRP, and hepcidin as compared to patients without anemia and the control group.

The concentration of ferritin, CRP, and hepcidin negatively correlates with hemoglobin levels in patients with COPD.

\section{REFERENCES}

1. Attaran D, Khajedalouee M, Ahmadi F et al. Anemia in COPD patients and its relation to serum levels of erythropoietin. Tanaffos. 2009;8(2):11-16.

2. Azza A, Hassan A, Said A et al. Relationship between hepcidin, ferritin and C-reactive protein in hemodialysis patients. The Egyptian Journal of Hospital Medicine. 2017;69(2):1786-1793.
3. Bryan J, Jacqueline M, Olivier D et al. Investigation of the role of interleukin- 6 and hepcidin antimicrobial peptide in the development of anemia with age. Hematologica. 2013;98(10):1633-1640.

4. Fidan $\mathrm{A}$, Tokmak $\mathrm{M}$, Kiral $\mathrm{N}$ et al Anemia in COPD and related factors. Chest. 2010;138:457.

5. Global Initiative for Chronic Obstructive Lung Disease. Global strategy for the diagnosis, management and prevention of chronic obstructive pulmonary disease. NHLBI/WHO workshop repot Updated 2018. Available at: www.goldcopd.com.

6. Hohaus S, Massini G, Giachelia M. Anemia in Hodgkin's lymphoma: The role of interleukin-6 and hepcidin. J ClinOncol. 2010;28:2538.

7. John M, Hoernig S, DoehnerW et al. Anemia and inflammation in COPD. Chest. 2005;127:825-829.

8. Kemna E, Pickkers $\mathrm{P}$, Nemeth $\mathrm{E}$ et al. Time-course analysis of hepcidin, serum iron, and plasma cytokine levels in humans injected with LPS. Blood. 2018;106(5):1864-1866.

9. Miller J, Edwards LD, Agusti A et al. Comorbidity, systemic inflammation and outcomes in the ECLIPSE cohort. Respir Med 2013; 107(9):1376-1384.

10. Nemeth $E$, Valore $V$, Territo $M$ et al. Hepcidin a putative mediator of anemia of inflammation is a type II acutephase protein. Blood. 2003;101:2461-2463.

11. Parveen S, Rangreze I, Ahmad SN et al. Prevalence of anemia in patients with COPD and its potential impact on morbidity of COPD patients. Int J Clin Med. 2014;5:452-8.

12. Pirotte M, Guiot J, Beguin Y et al. Anemia in patients with severe chronic obstructive pulmonary disease, a comorbity more common than previously thought. Rev Med Liege. 2016.Nov;71(11):488-494.

13. Queiroz D, Rocha A, Melo F et al. Increased gastric IL-1 $\beta$ concentration and iron deficiency parameters in H. pylori infected children. PLOS ONE. 2013;8(2).

14. Steinbicker A, Sachidanandan C, Vonner A et al. Inhibition of bone morphogenetic protein signaling attenuates anemia associated with inflammation. Blood. 2011 May 5;117(18):4915-23.

15. Tilg H, Ulmer $\mathrm{H}$, Kaser A et al. Role of IL-10 for induction of anemia during inflammation.J Immunol. 2002;169:2204.

16. Weiss $G$. Pathogenesis and treatment of anemia of chronic disease. Blood Rev. 2002;16:87-96.

\section{ORCID and contributionship:}

Anna V. Kovchun - 0000-0002-5856-8323 A,B,C,D,F

Vladyslav A. Smiianov - 0000-0001-8164-9706 E,F

Nataliia G. Kuchma - 0000-0003-0036-4915 C,E

Vladyslava V. Kachkovska - 0000-0002-9563-5425 A,E,F

Lyudmyla N. Prystupa - 0000-0002-6454-9831 A,B,D,E,F 
Conflicts of interest:

Authors declare no conflict of interest.

\section{CORRESPONDING AUTHOR}

Vladyslav A. Smiianov

Sumy State University

1 Sanatorna Str., 40018, Sumy, Ukraine

e-mail:v.smiyanov@med.sumdu.edu.ua

Received: 03.09.2019

Accepted: 30.12.2019

A - Work concept and design, B - Data collection and analysis, C - Responsibility for statistical analysis,

D-Writing the article, E-Critical review, $\mathbf{F}$ - Final approval of the article 